\title{
MARXISMUS UND BÜROKRATIE ${ }^{1}$
}

Wissenschaft von der Politik kann so wenig an die Stelle von Politik treten wie Literaturwissenschaft an die Stelle von Literatur. Aber, wie sich im Laufe der Zeit die Reflexion über Probleme der Literatur von dieser loslöste, so mußte auf einer bestimmten Stufe der Entwicklung des gesellschaftlichen und politischen Lebens auch die Reflexion über das Gemeinwesen und über politisches Handeln ein selbständiger Gegenstand der Forschung werden. Was vermag wissenschaftliche Besinnung über politische Probleme zu leisten? Vor allem kann sie - wie Wissenschaft überhaupt - dazu beitragen, die Elemente der uns umgebenden Wirklichkeit nüchtern und rational $\mathrm{zu}$ sehen und unsere politischen Urteile aus der Sphäre vorschneller, unbegründeter Meinungsbildung und gefühlsmässiger Voreingenommenheiten auf eine Ebene vernünftiger Erwägungen und begründbarer Erwartungen heben. Sie kann das politische Urteil klären und schärfen und damit die Voraussetzungen für politische Entscheidungen verbessern, diese selbst jedoch vermag sie weder zu ersetzen, noch zu bewirken. $\mathrm{Zu}$ einer solchen klärenden Besinnung sollen auch die vorstehenden Überlegungen über Marxismus und Bürokratie beitragen.

Unter Trotzkisten erzählte man sich, Lenin habe auf dem Sterbebett in tiefer Resignation ausgerufen: "was ist aus der Sowjetunion geworden? - ein bürokratisches Utopia!" Der Bericht ist sicher - wie die meisten Aussprüche sterbender Staatsmänner - eine Legende. Die Formel "bürokratisches Utopia" aber hat Lenin tatsächlich einmal - in dem Brief vom I 9.2. I 92 I an Gleb Maksimilianović Kržižanovskij gebraucht, um das Projekt einer "vollständigen, einheitlichen Planung" für die gesamte Union zu charakterisieren. ${ }^{2}$ Aber - so groß auch immer die Bemühungen Lenins in seinen letzten Lebensjahren waren, durch die "Arbeiter- und Bauerninspektion" und andere Maßnahmen, Auswüchse

1 Durch eine Anzahl Ergänzungen und Anmerkungen erweiterter Text der Antrittsvorlesung, die ich am 13.1 I. I959 an der Universität Tübingen gehalten habe.

2 V. I. Lenin, Sočinenija, 4. Aufl. Moskau 1952, t. 35 P. 4 O5. 
der Bürokratie zu bekämpfen - an der Tatsache, daß die Sowjetunion heute eine büroktatische Herrschaftsordnung darstellt, kann nur noch ein blinder Anhänger dieses Systems zweifeln.

Diese Tatsache steht aber in einem eigenartigen und frappierenden Gegensatz zu der äusserst scharfen Bürokratiekritik, die sich bei Marx und Engels von ihren frühesten Schriften angefangen bis an ihr Lebensende findet und die nach ihrem Tode nicht nur von der mittelund westeuropäischen Sozialdemokratie, sondern auch von der Partei Lenins aufgegriffen wurde. Wir haben hier - womöglich noch krasser als auf anderen Gebieten - einen Widerspruch, wie er für das Verhältnis von marxististischer Theorie und sowjetischer Praxis charakteristisch ist. Meine Ausführungen wollen einen Beitrag zur Erklärung dieses Widerspruchs leisten.

\section{I}

In einem I 858 für die New York Daily Tribune verfassten Artikel schildert Karl Marx die im Gegensatz zu den verfassungsmässigen Freiheitsrechten stehende Wirkung der preußischen Bürokratie wie folgt: „Bei jedem Schritt, selbst bei einer einfachen Ortsveränderung, tritt die allmächtige Bürokratie in Aktion, diese zweite Vorsehung preußischer Herkunft. Man kann weder leben noch sterben, weder heiraten, Briefe schreiben, denken, drucken, sich Geschäften widmen, lehren oder belehrt werden, eine Versammlung einberufen, eine Fabrik bauen, auswandern, noch überhaupt irgend etwas tun ohne, obrigkeitliche Erlaubnis" ". ${ }^{1}$ Dieses Plädoyer gegen die Bürokratie steht bei Marx keineswegs isoliert, und auch wenn man seine Schärfe und die Wahl der Beispiele zum Teil als eine Akkomodation an die Leserschaft der New Yorker Zeitung auffassen könnte, dürfte das Zitat im wesentlichen die Auffassung Marxens richtig wiedergeben. Diese Einstellung ist auch nicht weiter verwunderlich, wenn man sich daran erinnert, daß Karl Marx aus einer liberalen rheinischen Bürgerfamilie hervorgegangen ist und seine politische Laufbahn als Mitarbeiter und schließlich Redakteur eines linksbürgerlichen Kölner Blattes begann. Im August I 848 schrieb er in der „Neuen Rheinischen Zeitung": „Wie sehr die ,festgeregelten' und ,streng gehandhabten' Maßregeln der löblichen preußischen Bürokratie das ganze gesellschaftliche Leben, die industrielle und ackerbauende Produktion, den Handelsverkehr, den Bergbau, kurz alle gesellschaftlichen Beziehungen ohne Ausnahme ,störten', davon wissen... ganz besonders wir Rheinländer wunderbare Dinge zu erzählen". ${ }^{2}$ Es war also nicht besonders er-

\footnotetext{
1 Zit. nach Marx-Engels-Lenin-Stalin, Zur deutschen Geschichte, Berlin 1953/54, vol. II, p. 655.

${ }^{2}$ Zit. nach Marx-Engels-Lenin-Stalin, Zur deutschen Geschichte, 1.c., vol. II, p. 266.
} 
staunlich, daß die beiden Rheinländer Marx und Engels die preußische Bürokratie von vornherein in einem dunklen Lichte sahen und ihr späterer Aufenthalt in einem Lande obne derartige Bürokratie trug kaum dazu bei, ihre ursprüngliche Auffassung zu ändern.

Aber Marx und Engels wären nicht die historischen Denker, die sie sind, wenn sie auf dem Standpunkt stünden, Bürokratie sei gleich Bürokratie. Wenn sie auch nirgends im Zusammenhang eine Geschichte der Entwicklung und des Funktionswandels der Bürokratie gegeben haben, so kann man doch ihre zahlreichen Äusserungen mühelos zu einer Skizze einer derartigen Entwicklungsgeschichte zusammenfassen. Ich begnüge mich mit einem Hinweis auf die charakteristischsten Etappen.

Entstanden ist die europäische Bürokratie ,in der Zeit der absoluten Monarchie, beim Verfall des Feudalwesens, den sie beschleunigen half”. ${ }^{1}$ Damals hatte sie - nach Marx - eine durchaus „fortschrittliche”, nämlich die Entwicklung der bürgerlichen Gesellschaft beschleunigende, Wirkung. Der bürokratische Staat trat an die Stelle der ,widerstreitenden mittelalterlichen Machtvollkommenheiten" und verwandelte sie, in den geregelten Plan einer Staatsmacht, deren Arbeit fabrikmäßig geteilt und zentralisiert ist". ${ }^{2}$ Seine historische Aufgabe bestand nach Marx in der Aufhebung der Zwangskorporation (Zünfte, Gilden usw.) und der politischen Nivellierung der Staatsbürgerschaft. "Wo die Bürokratie neues Prinzip ist,... kämpft sie gegen die Korporationen" stellt Marx schon 1843 in seinem Manuskript zur Kritik des Hegelschen Staatsrechts fest. ${ }^{3}$ Aber mit dieser Funktion ist die nützliche Leistung der absolutistischen Bürokratie auch schon beendet. Kaum hat sie diese erfüllt, da verwandelt sie sich bereits in eine die Entwicklung hemmende Institution. In der Sprache des historischen Materialismus ausgedrückt: Absolutismus wie Bürokratie waren nur die zunächst unentbehrlichen Mittel, deren sich das Bürgertum (unbewußt) bediente, um die Fesseln der Feudalgesellschaft abzustreifen und die Gesellschaft der freien Konkurrenz durchzusetzen. Sobald aber das, was Marx I 843 „das wirkliche Staatsleben" nannte, erwacht und „die bürgerliche Gesellschaft sich von den Korporationen aus eignem Vernunfttrieb befreit", 4 geht die Bürokratie daran, die Korporationen wiederherzustellen. Mit dem Entstehen einer mächtigen demokratischen Bewegung, die von sich aus Gleichheitsforderungen stellt, fühlt sich die Bürokratie in ihrem politischen Privilegium bedroht und

\footnotetext{
1 Karl Marx, Der achtzehnte Brumaire des Louis Bonaparte, Berlin 1946, p. 102

2 l.c., p. I03.

${ }^{3}$ Marx-Engels, Werke, Berlin 1956sq., vol I, p. 247.

4.c., p. 247.
} 
versucht, die Herstellung der von ihr ursprünglich selbst geförderten Homogenität der Staatsbürgerschaft zu hintertreiben. Die Bürokratie muß „die imaginäre Allgemeinheit des besonderen Interesses, den Korporationsgeist, beschützen, um die imaginäre Besonderbeit des allgemeinen Interesses, ihren eignen Geist, zu erhalten". ${ }^{1}$ Mit anderen Worten: die Bürokratie wird zum erklärten Feind der Demokratie und als solcher wird sie auch von Karl Marx in seiner Kritik des Hegelschen Staatsrechts verurteilt. Bürokratie das ist für Marx nicht einfach die Gesamtheit beamteter Verwaltungsbehörden, sondern - wie sie ja auch in der Hegelschen Verherrlichung dargestellt wird - die Herrschaft einer Kaste, die sich auf Grund ihrer höheren Bildung die Interpretation des Staatsinteresses (des „Gemeinwillens”) als Monopol vorbehält, ähnlich wie sich Zünfte und Gilden bestimmte Produktions- und Verkehrszweige vorbehielten. Wenn daher auch die Bürokratie Zunftzwang und Standesunterschiede aufheben kann, soweit sie mit dem von ihr interpretierten Staatsinteresse in Konflikt geraten, so kann sie doch nicht dulden, daß ganz allgemein jedes soziale Monopol - und damit auch ihr eigenes - im Namen demokratischer Prinzipien angegriffen wird. Die Bürokratie behauptet vielmehr (durch den Mund Hegels, der - wie Marx sagt - ihr ideologisches Selbstverständnis naiv zum Ausdruck bringt) „das Staatswesen, das spirituelle Wesen der Gesellschaft in ihrem Besitz zu haben", ${ }^{2}$ ihr ,allgemeiner Geist ist daher das Mysterium, innerhalb ihrer selbst durch die Hierarchie, nach außen als geschlossene Korporation bewahrt" (l.c.). Hieraus aber folgt, $\mathrm{da} B$ „die Autorität... das Prinzip ibres Wissens, die Vergötterung der Autorität ihre Gesinnung" (l.c.) ist. Die unbezweifelbare Autorität des Vorgesetzten steht der ebenso fraglosen Inkompetenz des beschränkten Untertanenverstandes gegenüber. Der Eigenbewegung der bürgerlichen Gesellschaft wie ihrer Individuen wird prinzipiell mißtraut, die Welt erscheint als ein „bloßes Objekt bürokratischer Behandlung”, ${ }^{3}$ der Wille der Bürokratie als „,causa prima”.

Hegel hat in seiner Rechtsphilosophie versucht, den Beamtenstaat mit dem Gedanken der Demokratie zu versöhnen und sich hierzu vor allem darauf berufen, da $\beta$ ja jeder Bürger - vorausgesetzt, daß er die entsprechenden Eigenschaften entwickelt, - Beamter werden könne. Für diese Argumentation hat Marx nur Spott. In gleicher Weise könne man ja behaupten, im Katholizismus bestehe kein Gegensatz von Priestern und Laien, da jedem Laien freistünde Priester zu werden. „Daß jeder die Möglichkeit hat, das Recht einer anderen Sphäre zu erwerben beweise nur, daß seine eigene Sphäre nicht die Wirklichkeit

\footnotetext{
1 l.c., p. 248.

2 l.c., p. 249.

${ }^{3}$ l.c., p. 250.
} 
dieses Rechtes ist". ${ }^{1}$ In einer Demokratie dagegen „handele es sich nicht um die Möglichkeit jedes Bürgers, sich dem allgemeinen als einem besonderen Stand zu widmen, sondern um die Fähigkeit des allgemeinen Standes wirklich allgemein, d.h. der Stand jedes Bürgers zu sein" (l.c.). Die Examina, von deren Bestehen die Zulassung zum allgemeinen Stand abhängig gemacht wird, erscheinen ihm vom demokratischen Standpunkt aus als ebenso verkehrt. In einem ,vernünftigen Staat gehöre nämlich eher ein Examen dazu, Schuster zu werden, als exekutiver Staatsbeamter", denn ohne Fertigkeit in der Schusterei könne man sehr wohl ein guter Staatsbürger und sozialer Mensch sein, nicht aber ohne das ,nötige Staatswissen”. Das Examen der Beamten sei „nichts als eine Freimaurerformel, die gesetzliche Anerkennung des staatsbürgerlichen Wissens als eines Privilegiums", „die bürokratische Taufe des Wissens, die offizielle Anerkennung von der Transsubstantiation des profanen Wissens in das heilige", und in Parenthese fügt der fünfunzwangzigjährige Marx mokant hinzu: ,es versteht sich bei jedem Examen von selbst, daß der Examinator alles weiß"(l.c.). Offensichtlich macht Marx hier keinen Unterschied zwischen dem Verwaltungsbeamten im modernen Sinne und dem eigentlichen Politiker. Denn, obwohl er in dem eben zitierten Passus selbst von ,exekutiven Staatsbeamten” gesprochen hatte, fügt er die Bemerkung hinzu „,man hört nicht, daß die griechischen oder römischen Staatsleute Examina abgelegt" hätten, vergleicht also ungerechtfertigter Weise die antiken Staatsmänner mit den zeitgenössischen Beamten. Wie sehr übrigens das politische Denken von Marx und Engels - nicht nur in diesem Punkt - von dem antiken Polisideal beeinflußt ist, geht aus einer Analyse fast sämtlicher politischer Kategorien hervor, die sie benützen. Marx scheint 1843 die Tatsache zu ignorieren, daß - wenigstens in parlamentarischen Staaten - an der Spitze der Bürokratie politische „Beamte" zu stehen pflegen, von denen kein Examen gefordert wird und die die Verantwortung für die politischen Entscheidungen gegenüber dem Parlament (oder auch dem Monarchen) zu tragen haben. Das System der ministeriellen Verantwortlichkeit lernte Marx allerdings später in England kennen und beurteilte von daher auch die preußische Entwicklung: „die Frage der ministeriellen Verantwortung hat in Preußen... außergewöhnliches Gewicht, weil sie faktisch die Verantwortlicbkeit der Bürokratie bedeutet"2. Leider lege jedoch die preußische Verfassung nicht fest, wem die Minister verantwortlich seien und damit werde wohl alles beim alten bleiben. Die eifrigsten Bestrebungen des bürgerlichen Ehrgeizes in Preußen seien jedoch darauf ge-

\footnotetext{
1 1.c., p. 253.

${ }^{2}$ Marx-Engels-Lenin-Stalin, Zur deutschen Geschichte, 1.c., vol. II, p. 658.
} 
richtet "die Ministerposten in Preise zu verwandeln, die bei parlamentarischen Turnieren qu gewinnen sind". ${ }^{1}$

So wenig Marx zwischen der politischen Spitze der Bürokratie und der nachgeordneten Verwaltung unterschied, wie aus dem schiefen Vergleich mit den antiken Staatsmännern hervorging, so wenig deutlich wird bei ihm der Unterschied zwischen einer Bürokratie, die auf eigne Faust (unkontrolliert) herrscht und dem durch Parlament und öffentliche Meinung kontrollierten Verwaltungsapparat. Nur einmal heißt es bei ihm: „Unter der absoluten (französischen) Monarchie, während der ersten Revolution, unter Napoléon, war die Bürokratie nur das Mittel, die Klassenherrschaft der Bourgeoisie vorzubereiten... Unter... der parlamentarischen Republik war sie das Instrument der berrschenden Klasse, so sehr sie auch nach Eigenmacht strebte". ${ }^{2}$ Bis zu Napoléon hin erscheint ihm also die Bürokratie als herrschende Kaste, die die künftige Klassenherrschaft der Bourgeoisie vorbereitet, später als ein bloßes abhängiges Mittel in der Hand der Bourgeoisie. Ein Mittel freilich, das seiner Überzeugung nach die Tendenz hatte, erneut sich zu verselbständigen und auf eigne Faust zu herrschen. Nur in England und Amerika fehlte diese Gefahr, weil es dort keiner absolutistischen Bürokratie bedurft hatte, um der Herrschaft des Bürgertums gegenüber dem Feudalismus zum Durchbruch zu verhelfen.

\section{II}

Eine Wiederherstellung der unumschränkten Herrschaft der bürokratischen Exekutive empfanden Marx und Engels als das Hauptcharakteristikum des Bonapartismus, und zwar sowohl in seiner französischen wie in seiner deutschen (preußischen) Variante. Die Erklärung dieses politischen Phänomens konnte für sie nur in seiner Zurückführung auf spezifische Klassenverbältnisse bestehen und in der Tat liegen sogar mehrere derartiger Erklärungsversuche vor, denen wir uns jetzt zuwenden wollen.

Die erste Erklärung stammt aus dem Jahre i 8 5 I/ 52 von Karl Marx. In seiner Schrift „der I8. Brumaire des Louis Bonaparte" meint er, „die Staatsmaschine habe sich der bürgerlichen Gesellschaft gegenüber jetzt so befestigt, daß an ihrer Spitze" ein Louis Bonaparte genügte. Aber die Stärke des bürokratischen Apparates ist keine absolute Größe, sondern muß in Relation zur Schwäche der bürgerlichen Gesellschaft gesehen werden. Auch diese Staatsgewalt ,schwebt nicht in der Luft. Bonaparte vertritt eine Klasse, und zwar die zahlreichste der franzö-

1 1.c., p. 658 .

${ }^{2}$ Karl Marx, Der achtzehnte Brumaire, l.c., p. Iо3 f. 
sischen Gesellschaft, die Parzellenbauern". ${ }^{1}$ Das kleinbürgerliche französische Bauerntum bildet also - nach Marx - die Klassenbasis für die Herrschaft des bürokratischen Apparates mit Louis Bonaparte an der Spitze. Der Grund hierfür liegt in der politischen Ohnmacht und $\mathrm{Zu}$ rückgebliebenheit dieser Bevölkerungsschicht, die zwar ,an sich" bereits eine Klasse bildet, aber auf Grund des Fehlens jeder Verbindung unter ihren Angehörigen außerstande ist, zu einer eignen „politischen Organisation” zu gelangen und damit sich in eine „Klasse für sich” zu verwandeln. Als eine gleichsam unbewußte Klasse vermag sie daher auch nicht „ihre Klasseninteressen im eignen Namen, sei es durch ein Parlament, geltend zu machen”. Die Parzellenbauern „können sich nicht vertreten, sie müssen vertreten werden. Ibr Vertreter muß zugleich als ibr Herr, als eine Autorität über ibnen erscheinen, als eine unumschränkte Regierungsgewalt, die sie vor den anderen Klassen beschützt und ibnen von oben Regen und Sonnenschein schickt. Der politische Einfluß der Parzellenbauern findet also darin seinen letzten Ausdruck, daß die Exekutivgewalt sich die Gesellschaft unterordnet". ${ }^{2}$ Im Grunde liegt also gegenüber der kleinbürgerlichen Mehrheit der Bevölkerung das gleiche Verhältnis vor, das unterm Absolutismus im Verhältnis zur großbürgerlichen Minderheit bestand, die seinerzeit auch noch nicht im eignen Namen zu herrschen vermochte, sich von der Bürokratie des Absolutismus „vertreten” lassen mußte. Daß es aber gerade Louis Bonaparte war, der sich an die Spitze der verselbständigten Exekutive setzen konnte, das verdankte er in erster Linie dem Klang seines Namens und der Erinnerung, welche die Parzellenbauern mit ihm verbanden. Die politische Zurückgebliebenheit der Bauern war aber - wenigstens teilweise - durch die BourgeoisRegierungen selbst verschuldet, die im Kampf zwischen dem aufklärerischen Schulmeister und dem traditionalistischen Pfarrer den letzteren unterstützt und die dörfliche Selbstverwaltung der Herrschaft der Präfekten ausgeliefert hatten. Gegen Bauernaufstände aber waren von den gleichen Bourgeois-Regierungen Belagerungszustand und Exekutionen angeordnet worden. Die Bourgeoisie selbst „hat (also) den Imperialismus der Bauernklasse gewaltsam befestigt, sie hielt die $\mathrm{Zu}$ stände fest, die die Geburtsstätte dieser Bauernreligion bilden."

Ganz anders erklärt Engels 1872 in seiner Schrift ,zur Wohnungsfrage" die Entstehung des Bonapartismus in Preußen: ,Wir finden also hier neben der Grundbedingung der alten absolutistischen Monarchie: dem Gleichgewicht zwischen Grundadel und Bourgeoisie, die Grundbedingung des modernen Bonapartismus: das Gleichgewicht zwischen Bourgeoisie und Proletariat”. In beiden Fällen aber liege die ,wirkliche

1 l.c., p. 104.

2 l.c., p. Ios. 
Regierungsgewalt in den Händen einer besonderen Offiziers- und Beamtenkaste..." ' Die Verselbständigung der Bürokratie ist also hier die Folge eines labilen Klassengleichgewichts, sie tritt gleichsam als „lachender Dritter" gegenüber den Klassen auf. Gemeinsam an der Erklärung die Marx I $85 \mathrm{I}$ und Engels 1872 gab ist nur, daß in beiden Fällen die bürgerliche Gesellschaft oder ihre stärkste Klasse zu scbwach ist, um sich die bürokratische Exekutive unterzuordnen. In dem einen Fall, weil es ihr an dem nötigen Zusammenhalt und der erforderlichen Bewußtheit fehlt, im anderen, weil sich die beiden Hauptklassen gegenseitig paralysieren.

In einem Brief an Marx hat Engels aber auch eine noch weitgehendere These aufgestellt, der zufolge die Bourgeoisie überhaupt (wenigstens auf dem Kontinent) außerstande ist, selbst direkt zu herrschen: „Der Bonapartismus ist doch die wahre Religion der modernen Bourgeoisie. Es wird mir immer klarer, daß die Bourgeoisie nicht das Zeug hat, selbst direkt zu herrschen und daß daher, wo nicht eine Oligarchie, wie hier in England, es übernehmen kann, Staat und Gesellschaft... im Interesse der Bourgeoisie zu leiten, eine bonapartistische Halbdiktatur die normale Form ist; die großen materiellen Interessen der Bourgeoisie führt sie durch, selbst gegen die Bourgeoisie, lässt ihr aber keinen Teil an der Herrschaft selbst".2

Wir haben damit drei historische Erscheinungsformen der Bürokratie kennengelernt, die - wenn auch in unterschiedlicher Ausführlichkeit von Marx und Engels beschrieben wurden:

I. Die Bürokratie des Absolutismus, die als ein fortschrittliches Instrument der Nivellierung der Ständegesellschaft und für die Beseitigung der Korporationen angesehen wurde.

2. eine der - im Parlament zum Ausdruck kommenden - Bourgeois-

1 Marx-Engels, Ausgew. Schriften in 2 Bänden, Berlin 1953, vol. I, p. 574. Vgl. auch Der Ursprung der Familie des Privateigentums und des Staates, 1.c., vol. II, p. 298. Dort heißt es: „Ausnahmsweise indes kommen Perioden vor, wo die kämpfenden Klassen einander so nahe das Gleichgewicht halten, daß die Staatsgewalt als scheinbare Vermittlerin momentan eine gewisse Selbständigkeit gegenüber beiden erhält. So die absolute Monarchic des 17. u. 18. Jh., die Adel und Bürgertum gegeneinander balanciert; so der Bonapartismus des ersten und namentlich des zweiten französischen Kaiserreichs, der das Proletariat gegen die Bourgeoisie und die Bourgeoisie gegen das Proletariat ausspielte. Die neueste Leistung in dieser Art... ist das neue Deutsche Reich Bismarckscher Nation: hier werden Kapitalisten und Arbeiter gegeneinander balanciert und gleichmäßig geprellt zum Besten der verkommenen preußischen Krautjunker". Det österreichische Sozialdemokrat Otto Bauer hat in seinem Buch Die oesterreichische Revolution (Wien 1923) an Engels anknüpfend eine bestimmte Periode der Nachkriegszeit in Österreich gleichfalls als „Zeit der Gleichgewichte der Klassenkräfte" charakterisiert, nur mit dem Unterschied, daß jetzt das Proletariat selbst einen gewissen Anteil an der Ausübung der Regierungsgewalt hatte. 2 Brief vom 13.4.1 866 - zit. nach K. Marx-F. Engels, Ausgew. Briefe, Berlin I953, p. 208. 
Herrschaft unterworfene, ,uneigentlicbe" Bürokratie, die vergeblich nach Eigenmacht strebt;

3. die bonapartistische Bürokratie, die auf Grund der Schwäche der bürgerlichen Gesellschaft oder ihrer stärksten Klasse sich erneut zum Herrn des Staates machen kann.

Da die unter 2. genannte „uneigentliche” Bürokratie nach der Überzeugung von Marx stets die Gefahr mit sich führte, wieder in eine echte bürokratische Herrschaft umzuschlagen, schien es ihm - wenigstens auf dem Kontinent - wünschenswert, eine politische Form zu entwickeln, die einen solchen bürokratischen Apparat von vornherein überflüssig macht. Das kritische Motiv hinter der Bürokratiekritik von Marx wie von Engels ist aber die liberale und demokratische Überzeugung, daßdiebürgerlicheGesellschaftüberdenStaatund die vereinigten Staatsbürger über die Exekutive herrschen sollten. Bei aller Feindschaft vor allem gegen den Wirtschafts-liberalismus standen Marx und Engels doch den bourgeoisen Liberalen näher als den konservativen Bürokraten. In diesem Punkt trennten sie sich radikal von Lasalle. Vom Standpunkt ihres Entwicklungsdenkens aus war die reine Bourgeoisherrschaft, die der Selbstbewegung der bürgerlichen Gesellschaft freie Bahn eröffnet, dem reglementierenden bürokratischen System vorzuziehen, selbst wenn dieses im Einzelfall zugunsten des Proletariats eingreifen sollte.

Solange Marx und Engels daher primär die kontinentalen Verhältnisse im Auge hatten, weil die englische Arbeiterklasse noch wenig Anzeichen einer selbständigen politischen Bewegung gab, waren sie der Meinung, daß die proletarische Revolution vor allem den bürokratischen Staatsapparat zerschlagen müsse, um die Macht der politischorganisierten Arbeiterklasse an seine Stelle zu setzen. Der bestehende Verwaltungsapparat erschien ihnen als so eng mit der bürgerlichen (und kleinbürgerlichen) Klasse verbunden, daß er nicht zum Werkzeug einer proletarischen politischen Gewalt gemacht werden könnte, aber auch an den Aufbau eines neuen, dem Proletariat dienenden Apparates wurde nicht gedacht, sondern vielmehr an eine solche Form der Verwaltung, bei der keinerlei beamteter Apparat entsteht und die politische Macht unmittelbar von den gewählten Volksvertretern in Einzelmassnahmen umgesetzt wird.

\section{III}

In der klassenlosen Gesellschaft, die von Marx als Endphase der sozialen Entwicklung angenommen wird, fällt mit dem Staat auch das Problem des Bürokratie dahin. Es wird dann keinerlei politische Herrschaft des Menschen über den Menschen mehr geben und - nach 
einem Wort von Engels, das auf ältere Quellen zurückgeht, „die Verwaltung von Sachen an die Stelle der Herrschaft über Personen" treten. $\mathrm{Da}$ es freilich auch in dieser staatslosen Gesellschaft noch ein gewisses $\mathrm{Ma}$ von Über- und Unterordnung geben müsse hat Marx im „Kapital" wiederholt anerkannt, nur seien das eben rein sachlich bedingte, rational begründbare Verhältnisse, von denen er offenbar annahm, daß sie mit völliger Freiwilligkeit vereinbar sein würden. ${ }^{1}$ Anders liegt der Fall bei dem Übergangsstadium, das zwischen dem kapitalistischen Staat und der vollendeten klassenlosen Gesellschaft („Kommunismus”) liegt. Hier gibt es noch einen Staat, dessen „,Klassenwesen” von Marx als "Diktatur des Proletariats” bezeichnet wird. Über die „politische Form” dieser Diktatur war sich Marx lange Zeit im unklaren, bis er sie endlich $187 \mathrm{I}$ in der Pariser Kommune-Verfassung gefunden zu haben glaubte. Mit der Glorifizierung der Pariser Kommune in seiner Schrift „Der Bürgerkrieg in Frankreich” schuf Marx den Mythos einer modernen bürokratielosen, direkten Demokratie, an den Lenin 1917 anknüpfen konnte, um die spontan entstandenen Arbeiter- und Bauern-Sowjets, die ihm aus taktischen Gründen nützlich erschienen, marxistisch zu legitimieren.

Bei der Charakterisierung der Kommune zählt Marx die folgenden vier Grundzüge auf:

I. Geht die Kommune aus allgemeinen Wahlen in den Pariser Bezirken hervor, wobei die gewählten Stadträte zugleich ihren Wählern verantwortlich und jederzeit durch sie absetzbar sein sollen. Schon hierin erblickte Marx einen wesentlichen Fortschritt gegenüber dem üblichen parlamentarischen System, das die Verantwortlichkeit des Abgeordneten nicht durch ein Abberufungsrecht der Wähler unterstreicht.

2. Soll die „Kommune nicht eine parlamentarische, sondern eine arbeitende Körperschaft sein, vollziehend und gesetzgebend zu gleicher Zeit". Die Gewaltenteilung wird damit auch formell aufgehoben. Das Organ des Volkswillens, die Kommune, begnügt sich nicht mit dem Erlass allgemeiner - gesetzlicher Bestimmungen, sondern wendet diese auch selbst - womöglich mit Hilfe von Vollzugsausschüssen - auf den Einzelfall an.

3. Sollen Polizei und Beamtenschaft ihrer politischen Eigenschaften entkleidet und in jederzeit absetzbare Organe der Kommune verwandelt werden, wobei ihre Gehälter die Höhe eines Arbeiterlohnes nicht überschreiten dürfen. Was also an Restbeständen des bürokratischen Apparates als unentbehrlich beibehalten wird, soll seinen ,politischen Charakter" verlieren und sich in einfache Lohnarbeiter verwandeln, die von der Kommune ernannt und entlassen werden können, ein Ver${ }^{1}$ Karl Marx, Das Kapital, Volksausgabe, Berlin r956, vol. III, pp. 418f. 
fahren, das sich Marx so einfach vorstellt, wie Einstellung und Entlassung von Fabrikdirektoren durch den Aufsichtsrat einer Aktiengesellschaft! Die Bestimmung über die Höchstgehälter soll vermutlich verhindern, daß Beamtenstellen zum Ziel ehrgeiziger Wünsche und zum Preis für Wahlhilfen u. dergl. würden. Dabei sollte jede Kommune in Frankreich völlig freie Hand haben. Marx hat also den föderalistischen Charakter dieser politischen Ordnung durchaus bejaht, was Lenin später energisch leugnete. Ausdrücklich heißt es: „Die öffentlichen Ämter hören auf, des Privateigentum der Handlanger der Zentralregierung zu sein. Nicht nur die städtische Verwaltung, sondern auch die ganze, bisher durch den Staat ausgeübte Initiative wurde in die Hände der Kommune gelegt". ${ }^{1}$

4. Sollen auch die "richterlichen Beamten gewählt, verantwortlich und absetzbar sein" (l.c.). Es fehlt freilich der Hinweis darauf, durch wen diese Richterwahl erfolgen soll. Man kann jedoch annehmen, daß Marx an die Wahl durch das Volk selbst gedacht hat, da er die Unabhängigkeit der Justiz in den bisherigen politischen Systemen als bloßen Schein bezeichnet, der nur dazu gedient habe, deren faktische „Unterwürfigkeit unter alle aufeinanderfolgenden Regierungen zu verdecken", woraus man schliessen könnte, daß ihm eine wirkliche Unabhängigkeit der Justiz von der Regierung (d.h. auch von der Kommune) wünschenswert erschien.

In diesem extrem demokratischen und föderalistischen KommuneStaat würde nach der Überzeugung von Marx die nationale Einheit Frankreichs auf neuer, freiwilliger Basis und in dauerhafterer Weise erhalten bleiben als unter den bisherigen Regimes. Für eine sich verselbständigende Bürokratie aber würde keine Chance mehr bestehen.

Wenn man sich fragt, worin das Hauptmotiv für Marxens Eintreten für dieKommune-Verfassunglag, so wirdmaninersterLinieauf seineFurcht vor der Bürokratie stossen, deren „fürchterlichen Parasiten-körper” keine der Revolutionen seit $1789 \mathrm{zu}$ brechen vermochte, sondern der nur immer stärker aus jeder dieser Umwälzungen hervorging. Als ein weiteres Motiv könnte aber vielleicht auch der Gedanke eine Rolle spielen, daß das parlamentarische System mit seiner Kontrolle der Bürokratie nur sinnvoll ist, wenn es eine Mehrheit von Parteien gibt, die sich wechselseitig kontrollieren. Da nun aber mit dem Sieg des Proletariats diese Pluralität dahinfallen würde, erschien auch die parlamentarische Demokratie als überholt und eine neue, den Umständen angemessenere Form der Volksherrschaft wäre in der Kommuneverfassung von dem politischen Instinkt der aufständischen Pariser Arbei-

${ }^{1}$ Karl Marx, Der Bürgerkrieg in Frankteich, zit. nach Marx-Engels, Ausgew. Schriften in zwei Bdn., vol. I, p. 49 I. 
ter „erfunden” worden. Dieser Gedankengang ist freilich, wenn er Marx unterstellt wird, nur eine Hypothese. Spätere Marxisten haben ihn dagegen zur Rechtfertigung ihrer Ablehnung der bürgerlichen, parlementarischen Demokratie wiederholt vorgebracht.

\section{IV}

Charakteristischerweise haben aber weder Engels noch andere Sozialdemokraten nach Marxens Tod an seiner These von der KommuneVerfassung als der geeignetsten politischen Form für den Übergang vom kapitalistischen Staat zur Diktatur des Proletariats festgehalten. Friedrich Engels stellt zunächst in seiner Kritik des Erfurter Programms von I 89I fest, daß in Ländern wie England und Amerika - aber erstaunlicherweise auch in Frankreich - ein friedlicher Übergang zum proletarischen Staat möglich ist, weil dort die Volksvertretungen die wirklichen Machthaber sind. Nach dem Zusammenbruch des Bonapartismus erschien ihm offensichtlich die Bürokratie nicht mehr als eine so bedrohliche Macht, daß ihre vorherige Zerschlagung zur Voraussetzung der proletarischen Machtergreifung gemachtwerden müßte. Er meint: „Man kann sich vorstellen, die alte Gesellschaft könnte friedlich in die neue hineinwachsen in Ländern, wo die Volksvertretung alle Macht in sich konzentriert, wo man verfassungsmässig tun kann, was man will, sobald man die Majorität des Volkes hinter sich hat; in demokratischen Republiken wie Frankreich und Amerika, in Monarchien wie England..." 1 Das gilt aber nicht für Deutschland,

\footnotetext{
${ }^{1}$ Friedrich Engels, Kritik des Erfurter Programms der SPD, zit. nach K. Marx, Kritik des Gothaer Programms, Berlin 1946, p. 75. Im gleichen Zusammenhang warnt Engels jedoch vor zu starker Zentralisierung: „Also einheitliche Republik. Aber nicht im Sinne der heutigen französischen, die weiter nichts ist, als das 1798 begründete Kaiserreich ohne den Kaiser. Von 1792-1 $79^{8}$ besaß jedes französische Departement, jede Gemeinde vollständige Selbstverwaltung nach amerikanischem Muster, und das müssen wir auch haben. Wie die Selbstverwaltung einzurichten ist und wie man ohne Bureaukratie fertig werden kann, das bewies uns Amerika und die erste französische Republik, und noch heute Australien, Kanada und die anderen englischen Kolonien..." (p. 78). Als Mindestforderung solle daher ins Programm gesetzt werden: ,, vollständige Selbstverwaltung in Provinz, Kreis und Gemeinde durch nach allgemeinem Stimmrecht gewählte Beamte. Abschaffung aller von Staats wegen ernannten Lokal- und Provinzialbehörden" (l.c.). Diese Selbstverwaltung soll aber keinesfalls zu einer Aufrechterhaltung der „Kleinstaaterei” beitragen. In Deutschland könne das Proletariat nur eine „unteilbare Republik” brauchen. „Die Föderativrepublik ist auf dem Riesengebiet der Vereinigten Staaten jetzt noch im ganzen eine Notwendigkeit... Sie wäre ein Fortschritt in England, wo vier Nationen auf den beiden Inseln wohnen... Sie ist in der kleinen Schweiz schon längst ein Hindernis geworden, erträglich nur, weil die Schweiz sich damit begnügt, ein rein passives Glied des europäischen Staatensystems zu zein. Für Deutschland wäre die föderalistische Verscbweizerung ein enormer Rückschritt..." (p. 77).
} 
"wo die Regierung fast allmächtig und der Reichstag und alle anderen Vertretungskörper ohne wirkliche Macht" sind. Hier müßte daher wenn nicht taktische Rücksichten dagegen sprächen - die Forderung nach der demokratischen Republik zum Programm der SPD gemacht werden, denn ,wenn etwas feststeht, so ist es dies, daß unsere Partei und die Arbeiterklasse nur zur Herrschaft kommen kann unter der Form der demokratischen Republik. Diese ist sogar die spezifische Form für die Diktatur des Proletariats, wie schon die grosse französische Revolution gezeigt hat..." Wenn man aber schon aus den erwähnten Gründen auf die Forderung der demokratischen Republik verzichten müsse, so solle doch auf jeden Fall ,die Forderung der Konzentration aller politischen Macht in den Händen der Volksvertretung" ins Programm aufgenommen werden. ${ }^{1}$

Noch deutlicher als Engels hat Eduard Bernstein sich von der Marxschen Verherrlichung der direkten Kommune-Demokratie distanziert. Dabei beruft er sich auf die Erfahrungen der englischen Gewerkschaften, wie sie in dem Buch von Sidney und Beatrice Webb über „Industrial Democracy” niedergelegt sind. „Die englischen Gewerkschaften”, schreibt Bernstein, ,haben mit der elementarsten Form der Selbstregierung begonnen und sich durch die Praxis überzeugen müssen, daß diese Form auch nur für die elementarsten Organismen, für ganz kleine Lokalvereine passt. Sie haben, je mehr sie wuchsen, Schritt für Schritt auf gewisse Lieblingsideen des doktrinären Demokratismus (das gebundene Mandat, der unbezahlte Beamte, die machtlose Zentralvertretung) als ihre gedeihliche Entwicklung lähmend verzichten und dafür eine leistungsfähige Demokratie mit repräsentativen Versammlungen, bezahlten Beamten und bevollmächtigter Zentralleitung ausbilden gelernt. Dieses Stück Entwicklungsgeschichte der „gewerblichen Demokratie" ist ungemein lehrreich. Paßt auch nicht alles, was von den Gewerkschaften zutrifft, für die Einheiten der nationalen Verwaltungskörper, so trifft doch sehr vieles davon auch für sie zu". ${ }^{2}$ Eine vollziehende Zentralverwaltung sei schon auf Grund der Arbeitsteilung von einem gewissen räumlichen Umfang an unentbehrlich. Eduard Bernstein hielt es also für unmöglich, daß die politische Leitung eines modernen Gemeinwesens von Kommunen und ihren Organen übernommen wird. Dabei betont er freilich zugleich die wachsende Bedeutung der städtischen Selbstverwaltung als einer „Schule der Demokratie". An die Stelle der übersteigerten Marxschen Hoffnung auf eine vollständige Beseitigung zentraler Verwaltungskörper tritt also bei Bernstein deutlich die Forderung nach ihrer parlamentarischen

1 1.c., p. 77 .

2 Eduard Bernstein, Die Voraussetzungen des Sozialismus und die Aufgaben der Sozialdemokratie, Stuttgart 1920, p. I95 (I. Aufl. I899). 
Kontrolle. In noch stärkeren Maße als beim späten Engels haben sich hier die englischen Erfahrungen ausgewirkt.

Auch beim Exponenten der marxistischen Orthodoxie nach Engels' Tod, bei Karl Kautsky, finden sich ähnliche Gedanken. Er hat sogar in einem späten Werk ausdrücklich die Verwaltungsbehörden verteidigt und betont, daß sie nicht notwendig autokratische Institutionen sein müßten: „Nicht die Bürokratie als solche ist das Übel”, meint er, "wohl aber ihre Allmacht, ihre Pedanterie sowie das, was Engels-Marx als Kretinismus bezeichneten, die Neigung der Mitglieder einer besonderen Institution, ihre Bedeutung für den gesellschaftlichen Gesamtprozeß zu überschätzen... Der bürokratische Kretinismus wird hochgradig in der absoluten Monarchie, wo die Obrigkeit alles zu entscheiden... hat... ist aber nicht notwendig mit ihr verbunden." So sei etwa in England „die Bürokratie nicht allmächtig, dort wiedersetze sich die Masse der Bevölkerung jeder polizeilichen Bevormundung, und ihre kräftige Selbstbetätigung hindere bei den Bürokraten zwar nicht völlig das Aufkommen, wohl aber das Erstarken von Weltfremdheit, Pedanterie und Kretinismus". 1

Die neue Einschätzung von Bürokratie und Parlamentarismus bei allen genannten Theoretikern dürfte einerseits mit dem Zusammenbruch des Bonapartismus in Frankreich und zum anderen mit dem Erstarken der politischen Arbeiterbewegung in England sowie mit der Erwartung zusammenhängen, die Verhältnisse auf dem Kontinent würden sich allmählich den englischen (und amerikanischen) annähern. Endlich aber schien das gewaltige Wachstum der Stimmenzahl der deutschen Sozialdemokraten zu beweisen, daß selbst in dem ,junker-

1 Karl Kautsky, Materialistische Geschichtsauffassung, 1927, vol II, p. 460. Vgl. auch ders. Parlamentarismus u. Demokratie, Stuttgart I9I I (1. Aufl, I893). Dort erklärt K. u.a.: „Heute kann nur ein politisch Blinder noch behaupten, das Repräsentativsystem sichere auch unter der Herrschaft des allgemeinen Wahlrechts die Herrschaft der Bourgeoisie, und um diese zu stürzen, müsse man zunächst das Repräsentativsystem beseitigen. Jetzt schon beginnt es offenbar zu werden, daß ein wirklich parlamentarisches Regime ebensogut ein Werkzeug der Diktatur des Ptoletariats sein kann, als es ein Werkzeug der Diktatur der Bourgeoisie ist. Nicht das Repräsentativsystem zu beseitigen, sondern die Macht der Regierung gegenüber den Parlamenten zu brechen, gleichzeitig aber auch dem Proletariat zu diesen eine möglichst breite Bahn zu ebnen durch Gleichheit des Wahlrechts, gieichmässige Einteilung der Wahlkreise, Wahrung des Wahlgeheimnisses, kurze Parlamente, völlige Freiheit der Presse, der Versammlungen und der Vereine, vor allem aber durch Ausdehnung des Wahlrechts auf alle Staatsangehörigen, die das zwanzigste Lebensjahr erreicht haben, das ist eine der wichtigsten Aufgaben der Arbeiterklasse in ihrem Kampfe um die Erringung der politischen Macht"(pp. I 2 I f.). Schon zuvor, in seiner Schrift über das „Erfurter Programm” hatte Kautsky erklärt: „Die direkte Gesetzgebung durch das Volk kann, wenigstens in einem modernen Großstaat ... das Parlament nicht überflüssig machen, sie kann höchstens neben ihm in Einzelfällen zu seiner Korrigierung in Tätigkeit treten... Solange der moderne Großstaat besteht, wird der Schwerpunkt der politischen Tätigkeit stets in seinen Parlamenten liegen" (pp. 220-221). 
lich-bürokratischen" deutschen Reich die Möglichkeit einer friedlichen Machtergreifung näherrückte, für die eine Verfassungsänderung zugunsten einer echten parlamentarischen Demokratie als ausreichende Vorbedingung angesehen wurde.

Im Gegensatz zur herrschenden Auffassung der europäischen Sozialdemokratie und sogar im bewußten Gegensatz zu dem sonst als beinahe "unfehlbar" angesehenen Engels hat W.I. Lenin (zum ersten Male schon nach der Revolution von 1905 aber nachdrücklich und entscheidend erst) 1917 auf den Marxschen Kommune-Mythos zurückgegriffen, um die spontan entstandenen Sowjets als Organe einer revolutionären und proletarischen Demokratie marxistisch zu rechtfertigen. In seiner 1917 veröffentlichten politischen Hauptschrift „Staat und Revolution" konzentriert er zwar seine Hauptaufmerksamkeit auf die These von der Notwendigkeit der Zerschlagung des alten Staatsapparates, wobei er Marx höchst einseitig interpretiert, daneben finden sich hier jedoch fast vollzählig die von Marx hervorgehobenen Grundzüge der Pariser Kommuneverfassung als Forderungen für den aufzubauenden proletarischen Staat wieder. Diese Forderungen sind am prägnantesten zusammengefasst in der 5. der berühmten Aprilthesen von 1917:

„Keine parlamentarische Republik - von den Sowjets der Arbeiterdeputierten zu dieser zurückzukehren wäre ein Schritt rückwärts sondern eine Republik der Sowjets der Arbeiter-, Landarbeiter- und Bauerndeputierten im ganzen Lande von unten bis oben. Abschaffung der Polizei, der Armee, der Beamtenschaft. Entlohnung aller Beamten, die durchwegs wählbar und jederzeit absetzbar sein müssen, nicht über den Durchschnittslohn eines qualifizierten Arbeiters hinaus". ${ }^{1}$

Die Räte oder Sowjets erwiesen sich allerdings sehr bald als unfähig die zahlreichen politischen, wirtschaftlichen und militärischen Aufgaben zu erfüllen, die an den jungen Staat herantraten, sodaß wenig später ein neuer zentraler Staatsapparat entstand, in dem wohl auch ein Teil der ehemaligen Beamten Unterschlupf fand. Dieser Apparat wurde zwar faktisch weder von den dezentralisierten Sowjets noch von dem obersten Organ der Sowjets ausreichend kontrolliert, wohl aber von der Partei, die bald über einen ebenbürtigen und auf die Dauer sogar überlegenen Apparat verfügte. Solange nun diese beiden zentralistischen Machtapparate sich noch relativ unabhängig gegenüberstanden, blieb auch noch ein Minimum an Freiheit für die einzelnen Sowjetbürger gewahrt. Dieses mußte aber vollends verlorengehen mit der Verschmelzung der beiden Apparate, die sich unter Stalins Herrschaft rasch vollendete.

1 W. I. Lenin, Über den Parteiaufbau, eine Sammlung ausgewählter Aufsätze und Reden, Ost-Berlin 1958, p. 487. In diesem Band sind alle wichtigen Arbeiten Lenins zu Organisationsfragen vereinigt und bequem erreichbar gemacht. 
Lenin selbst hatte schon seit i 898 („Die Aufgaben der russ. Sozialdemokraten") und vor allem in seiner Schrift "Was tun?" (I 902) für den Aufbau der Partei mit bemerkenswerter Offenherzigkeit eine straff zentralistische und autoritäre Struktur gefordert. Diese Prinzipien erwiesen sich als weit realistischer als die Hoffnungen auf die Funktionsfähigkeit der direkten Sowjetdemokratie. Sie wurden dem künftigen Sowjetstaat zum Schicksal. Ich muß sie daher in aller gebotenen Kürze in Erinnerung bringen:

I. Da das Industrieproletariat aus eigner $\mathrm{Kraft}$ außerstande ist, sich das „politische Klassenbewußtsein” anzueignen, sondern auf einem tradeunionistischen Standpunkt verharrt, muß ihm dieses politische Bewußtsein von außen her beigebracht werden. Diese Aufgabe hat die Partei als die „klassenbewußte Avant-garde” des Proletariats zu übernehmen. Es ist daher verständlich, daß die ersten Parteimitglieder nicht selbst der Arbeiterklasse entstammen, sondern ehemalige Bourgeois oder Kleinbürger sind, die sich dem Proletariat angeschlossen haben. Mit ihnen vereinigen sich in der Partei diejenigen Arbeiter, die sich bereits das politische Klassenbewußtsein erworben haben zu einer homogenen Gemeinschaft, die keinerlei Unterschiede der Klassenherkunft mehr kennt.

2. Entsprechend ihrem Verhältnis zur Klasse hat die Partei die Doppelaufgabe das Proletariat

a) zu führen und

b) zu erziehen. Die Führung ist notwendig autoritär solange die Erziehung noch nicht zur Einsicht und zur Aneignung des politischen Klassenbewußtseins durch alle geführt hat. Die Partei kann zwar nur überreden aber sie darf und muß sich zu agitatorischen Zwecken auch vereinfachender Darstellungen und dem begrenzten Auffassungsvermögen der zeitgenössischen Arbeiter angepasster Losungen bedienen, die den Anforderungen der "Wissenschaft" nicht zu entsprechen brauchen.

3. Angesichts der zaristischen Autokratie und der Allgegenwart der Geheimpolizei ist an eine breite innerparteiliche Demokratie nicht zu denken. Die Partei ist aufzubauen als eine konspirative Elite. In ihr gilt das Gesetz militärischer Disziplin und streng geregelter Befehlsund Gehorsamsverhältnisse. Ihre Führungsgremien ergänzen sich durch Kooptation. ${ }^{1}$ Später wird jedoch (nach 1905) der sogenannte „demokratische Zentralismus” eingeführt, demzufolge die personelle Zusammensetzung der Führungsgremien der Partei auf demokratischem Wege, die politische Generallinie der Partei aber auf zentralistische Weise bestimmt wird. In einem im November I90s erschienenen Artikel „Über die Reorganisation der Partei” fordert Lenin den „Über-

1 W. I. Lenin, Brief an einen Genossen, Genf I 904, zit. nach dem Sammelband „Uber den Parteiaufbau", p. 126. 
gang zum Prinzip der Wählbarkeit", 1 und in einem Resolutionsentwurf vom März des folgenden Jahres schreibt er:

„... das Prinzip der Wählbarkeit muß in den Parteiorganisationen von unten bis oben durchgeführt werden; Abweichungen von diesem Prinzip, beispielsweise zweistufige Wahlen oder Kooptierung in gewählte Körperschaften usw. sind lediglich bei unüberwindlichen polizeilichen Hindernissen und in besonders vorgesehenen Ausnahmefällen zulässig. ${ }^{2}$

In einem Artikel aus dem Jahre 1907 geht Lenin sogar noch weiter und fordert bei schwerwiegenden politischen Entscheidungen der Parteiführung eine vorherige Befragung aller Mitglieder der Partei eine Art „Referendum”, wie er es selber nennt. „Besonders wichtige Fragen, solche die unmittelbar mit einer bestimmten Aktion der Massen selbst verbunden sind, müssen um der Demokratie willen nicht durch Entsendung von Vertretern, sondern durch die Befragung aller Parteimitglieder entschieden werden". ${ }^{3}$

Trotz derartiger demokratischer Äusserungen Lenins hat sich jedoch in seiner Partei in zunehmendem Masse der autoritäre Zentralismus durchgesetzt, der wohl zu Lebzeiten Lenins auf Grund des ungeheueren Ansehens, das sich Lenin zu verschaffen wußte, noch nicht in Konflikt mit dem tatsächlichen Willen der Parteimitglieder geriet, nach seinem Tode aber unter der sich herausbildenden Alleinherrschaft Stalins immer stärker totalitäre Züge annahm. Durch seine Organisationslehre hat Lenin dieser Entwicklung zum mindesten Vorschub geleistet. Nach der Beseitigung nicht nur der bürgerlichen, sondern auch der konkurrierenden proletarischen und bäuerlichen Parteien und nach Aufhebung der Pressefreiheit wurde die Partei Lenins faktisch zur Alleinherscherin des Landes. Innerhalb der Partei aber war es ein ganz kleiner Kreis von „Berufsrevolutionären”, der die politischen Entscheidungen traf. Dem Apparat wohnte eine bemerkenswerte Tendenz zur Herausstellung einer Führerpersönlichkeit inne, die von Trotzki und seinen Anhängern nicht zufällig mit dem Phänomen des Bonapartismus verglichen wurde.

Es war nur die Auswirkung eines einfachen Gesetzes der politischen Soziologie wenn sich der zentral gesteuerte, einheitliche Parteiapparat alsbald gegenüber den dezentralisierten, uneinheitlichen, unerfahrenen

1 W. I. Lenin, Uber die Reorganisation der Partei, November I go5, in der Zeitschrift Novaja Zizn, auch in dem Sammelband Úber den Parteiaufbau pp. 4I 2-42I sowie in den Gesammelten Werken, vol. X, pp. I3-22.

${ }^{2}$ W. I. Lenin, Taktische Plattform zum Vereinigungsparteitag der SDAPR ... Die Grundlagen der Organisation der Partei, 20.3.1906, veröffentlicht in Partijnye Izvestija, zit, nach dem Sammelband „Über den Parteiaufbau”, pp. 422-423.

${ }^{3}$ W. I. Lenin, Sozialdemokratie und Dumawahlen, Jan. I907, zit. nach dem Auszug im Sammelband über den Parteiaufbau, pp. 427-428. 
und ohnmächtigen Sowjets durchsetzte. Wenn auch nach der Theorie Lenins Partei und Sowjets einander ergänzen sollten, indem die Partei die Werktätigen erzog und schulte, um ihnen die richtige Wahrung ihrer Interessen in den Sowjets zu ermöglichen, so erwies sich doch bald die Methode des Anordnens und Verfügens von der Zentrale aus als praktikabler. Es gelang nicht, das Rätesystem am Leben zu erhalten.

Charakteristisch für die schon unmittelbar nach der Oktoberrevolution einsetzende Kritik am bürokratischen System ist aber die Verschiebung des kritischen Akzentes von der Institution auf eine psychische Haltung. Bereits I 904 schrieb Lenin: „Das Wort Bürokratismus kann ins Russische übersetzt werden mit dem Wort: Rangstreit. Bürokratismus bedeutet: die Interessen der Sache den Interessen der Karriere unterordnen, hinter den Posten her sein und die Arbeit links liegen lassen, sich um die Kooptation balgen, anstatt für die Idee zu kämpfen". ${ }^{1}$ In diesem Sinne kann natürlich jede noch so absolut herrschendende Bürokratie den „Bürokratismus” bekämpfen und die alte preußische Beamtenschaft könnte in dieser Hinsicht geradezu als Vorbild gelten.

\section{VI}

Die unter Stalin zur Reife gelangte neue bürokratische Herrschaftsordnung in der Sowjetunion kennzeichnet Leo Trotzki - in Erinnerung an die Engelsschen Analysen wie folgt: „Der Bonapartismus betritt die Bühne der Geschichte immer dann, wenn der scharfe Kampf zweier Lager die Staatsmacht gleichsam über die Nation erhebt und sie scheinbar von den Klassen völlig unabhängig macht... Das Stalinregime, das über die politisch atomisierte Gesellschaft hinausragt, sich auf Polizei und Offizierskorps stützt und keinerlei Kontrolle über sich duldet, ist deutlich eine Sorte Bonapartismus neuen Typs...”.2 Die

1 W. I. Lenin, Ein Schritt vorwärts, zwei Schritte zurück (1904), zit. nach Über den Parteiaufbau, p. 304.

2 L. Trotzki, Verratene Revolution, Zürich o.J. (1957), p. 269. Vgl. auch: „die immer aufdringlichere Vergottung Stalins bildet..... einen unerlässlichen Bestandteil des Regimes. Die Bürokratie braucht einen unantastbaren obersten Schiedsrichter, einen Ersten Konsul, wenn nicht einen Kaiser...”.,„Stalin ist die personifizierte Bürokratie, und das macht seine politische Persönlichkeit aus” (l.c.). „Die Geschichte ist Zeuge, daß sich der Bonapartismus mit dem allgemeinen und selbst geheimen Wahlrecht ausgezeichnet verträgt. Das demokratische Ritual des Bonapartismus ist das Plebiszit. Von Zeit zu Zeit wird den Bürgern die Frage vorgelegt: für oder gegen den Führer? Wobei der Abstimmende den Revolverlauf an der Schläfe fühlt. Seit den Zeiten Napoleons III., der heute wie ein provinzieller Dilettant aussieht, hat diese Technik eine ungeahnte Entwicklung erfahren. Die neue Sowjetverfassung, die den Bonapartismus auf plebiszitärer Grundlage errichtet, ist die echte Krönung des Systems" (p. 270.). - Eine umfassende Kritik des bürokratischen Systems des bolschewistischen Staates gibt - von einen „anarchistischen" Standpunkt George Spiro, Marxism and the bolshevik state, workers democratic world government versus national-bureaucratic soviet and capitalist Regimes, New York I951. 
beiden „Klassen”, deren Gleichgewicht die Voraussetzung für die absolute Herrschaft der bürokratischen Elite darstellt, sollten die Industriearbeiterschaft und das Bauerntum sein.

Noch einleuchtender erscheint mir aber eine Anwendung der Marxschen Bonapartismus-Theorie auf die Sowjetgesellschaft. Die Basis wäre dann nicht so sehr der Klassendualismus als vielmehr die Existenz einer als solcher führenden Klasse - der Parteielite - (oder auch der sowjetischen Oberschicht als solcher), die jedoch nicht imstande ist, im eignen Namen und durch spontane Vereinigung ihre politischen Interessen zu vertreten. Einer Klasse, deren Angehörige sich wenn auch aus anderen Gründen in einer ähnlichen Vereinzelung und individueller Ohnmacht befinden, wie einst die französischen Parzellenbauern. Diese Klasse könnte ebenfalls nicht anders „,vertreten werden" als indem ihr Vertreter zugleich als ihr Herr auftritt. Als der Exponent einer allmächtigen Exekutive, die zwar mehr oder weniger gut die Interessen der Klasse als ganzer berücksichtigt, aber keine Rücksicht auf die Bedürfnisse der Individuen nimmt. Mir scheint, daß dieses Modell ohne nennenswerte Einschränkung zumindest auf die Stalinära anwendbar ist. Der sogenannte „Persönlichkeitskult” erklärt sich damit zugleich als ein typisches Phänomen der Bürokratie, denn „die Autorität ist das Prinzip ihres Wissens und die Vergötterung der Autorität ihre Gesinnung". Der von Werner Hofmann erhobene Einwand gegen die Behauptung der Existenz einer neuen herrschenden Klasse in der Sowjetunion fiele damit zusammen: die Existenzunsicherheit der Angehörigen einer Klasse ist kein Beweis gegen die kollektive Herrschaft derselben. ${ }^{1}$

Georg Lukács hat in einem seiner wenigen politischen Aufsätze unter dem Titel „Volkstribun oder Bürokrat” den Gegensatz zwischen der Haltung des Apparatschik und der des Politikers oder Demagogen herausgearbeitet. ${ }^{2}$ Sicher ist diese Erkenntnis bei ihm auf Erinnerungen an die politische Soziologie Max Webers zurückzuführen. Denn Weber ist es ja gewesen, der mit Nachdruck auf die Ungeeignetheit von Verwaltungsbeamten für politische Aufgaben hingewiesen und namentlich auch aus diesem Grunde das Parlament als einen zweckmässigeren Ort politischer Führerauslese angesehen hat. In einem bürokratischen Herrschaftssystem ohne echtes parlamentarisches Leben

\footnotetext{
1 Werner Hofmann, Die Arbeitsverfassung der Sowjetunion, Volkswirtschaftliche Schriften, Heft 22, Duncker \& Humblot, Berlin 1 956. Vgl. auch die Rezension von Heinz Maus und mir in: Neue Politische Literatur, III. Jg. H. 1, pp. 56-66.

${ }^{2}$ Georg Lukács, Volkstribun oder Bürokrat (1940), zit. nach Karl Marx und F. Engels als Literaturhistoriker, Berlin I952, pp. x 275q. Vgl. Max Weber, Gesammelte Politische Schriften, München 1921, pp. 139sq.
} 
und ohne Parteienkampf wird es aber sehr schwer sein, politische Begabungen zu offenbaren und zu entwickeln. Mit der Konsolidierung der Sowjetunion tritt denn auch der Typus des politischen Kämpfers gegenüber dem des Apparatschik immer mehr in den Hintergrund. Das Fehlen politischer Begabungen in der Führung wurde freilich solange nicht als Mangel empfunden, als der eine mächtige Herr an der Spitze des Systems stand. Nach dem Tode Stalins aber bedurfte es einer längeren innenpolitischen Auseinandersetzung, bis sich aus der Schar höher plazierter Bürokraten der für die Führungsaufgabe qualifizierte Politiker Nikita Chruščev herauslösen konnte. An der Spitze des Systems muß immer ein Politiker stehen, an denen die Sowjetunion wahrscheinlich nicht reich ist, denn zum Volkstribun kann sich in diesen Ländern kaum jemand ausbilden, es sei denn in der lebensgefährlichen Auflehnung gegen die allmächtige Bürokratie. Es ist allerdings denkbar, daß Parteifunktionäre der unteren und mittlereren Stufen, die nicht durchwegs verwalten und befehlen können, sondern mitreißen und überzeugen müssen, ein gewisses $\mathrm{Maß}$ politischer Führerbegabung benötigen und entwickeln. Nikita Chruščev wäre dann als das erfolgreiche Produkt einer derartigen Karriere anzusprechen zumal in der ukrainischen KP, aus der er herstammt, auch unter Stalin das Einmannführerprinzip unbekannt war.

In der Kritik vieler Marxisten am bürokratischen Sowjetsystem sind vielfach die theoretischen Irrtümer wiederholt worden, die zu seiner Entstehung beigetragen haben. Die Polemik Trotzkis und seines Parteigängers Rakovskij gegen die Stalinsche Bürokratie vergisst, daß keine zentral gelenkte Planwirtschaft ohne beamteten Apparat auskommen kann und verkennt damit die teilweise Berechtigung der von Stalin vollendeten Bürokratisierung des Landes. Zumindest auf der Entwicklungsstufe, auf der sich die Sowjetunion damals befand, war sozialistische Planwirtschaft ohne Aufbau eines bürokratischen Herrschaftsapparates eine Utopie. In den späteren Schriften Trotzkis und seiner Anhänger finden sich aber schon Einsichten, die der wahren Wurzel des totalitären Systems der Sowjetunion näherkommen, und in der „verratenen Revolution” fordert Trotzki sogar ausdrücklich die Zulassung mehrerer sozialistischer Parteien, Pressefreiheit und eine funktionsfähige Kontrolle der staatlichen Behörden. ${ }^{1}$ Die Sowjetbürokratie wäre freilich die letzte, solche Forderungen freiwillig zu

${ }^{1}$ Leo Trotzki, a.a.O., pp. 259 ff. Vgl. auch zur Kritik der Trotzki-Gruppe an der Stalinschen Bürokratie den von der „Vierten Internationale” in Paris herausgebrachten Band „Les Bolchéviks contre Staline 1923-1928", der Trotzkis "Cours nouveau" und die berühmte Schrift von Christian Rakovsky, "Les dangers professionnels du pouvoir”, sowie die Plattform der Linksopposition von 1927 enthält. 
gewähren. Trotzki hält daher auch eine zweite - rein politische - Revolution in der Sowjetunion für unabdingbar.

Max Weber hat in „Wirtschaft und Gesellschaft" die wachsende Bürokratisierung als ein gleichmäßig alle Lebensgebiete umfassendes Entwicklungsgesetz der industriellen Gesellschaft nachgewiesen und betont, daß dieses Gesetz auch vor dem sozialistischen Staat nicht haltmachen werde. In dieser Perspektive kommen einige Autoren zur Behauptung einer weitgehenden Ähnlichkeit der Sozialstruktur in Ost und West und knüpfen hieran womöglich noch die Hoffnung auf einen Ausgleich durch allmähliche Anpassung. In diesem Zusammenhang ist die Arbeit von Helen Contas über die zwei Bürokratiebegriffe bei Max Weber verdienstvoll und klärend. ${ }^{1}$ Contas weist darauf hin, $\mathrm{da} ß$ Weber nicht immer genügend scharf $z$ wischen einer legal-rationalen und einer charismatischen Bürokratie unterscheide. Bei der ersteren handele es sich um einen bloßen Verwaltungsstab, der sich im allgemeinen selbst als bloßes Mittel für von außen gesetzte Zwecke begreift, im anderen um ein System, das sich als obersten Zweck versteht und aus der Veralltäglichung des einem Staatengründer anhaftenden Charismas entstand. Beispiele für derartige charismatische Bürokratien sind ihr das spätrömische und byzantinische Kaiserreich, die katholische Kirche und das Sowjetsystem. Weber habe sich aber geirrt, wenn er - von seinem liberalen Fortschrittsglauben verführt - die Entwicklung der charismatischen zu legal-rationalen Bürokratien erwartet habe. Alle derartigen charismatischen Bürokratien erwiesen sich im Gegenteil als äusserst zählebig und entwicklungsfeindlich.

Fassen wir also zusammen: Marx und Engels waren leidenschaftliche Gegner der Bürokratie. Sie verachteten die kontinentale Bourgeoisie weil sie nicht in der Lage war, im eignen Namen durch ein Parlament zu herrschen. Für die Übergangszeit zwischen Kapitalismus und vollendeter kommunistischer Gesellschaft erstrebte Marx - nach dem Muster der Pariser Kommune - eine Ausschaltung der Bürokratie durch direkte Volkswahl aller Verwaltungsbeamten und Richter in den Gemeinden, Limitierung der Gehälter auf das Niveau von Arbeitslöhnen und Einrichtung von zugleich gesetzgebenden und regierenden Körperschaften. Während Engels, Kautsky, Bernstein und andere Sozialdemokraten später diese Theorie wieder fallen liessen und in einer die Exekutive kontrollierenden parlamentarischen Demokratie die politische Form für die Übergangszeit erblickten, kehrte Lenin I9I7 zu Marx' Kommune-Thesen zurück. Die Sowjets erwiesen sich jedoch bald als unpraktikabel und gerieten in Konflikt mit der

1 Helen Contas, Max Webers two concepts of bureaucracy, in: American Journal of Sociology, LXIII, jan. 1958, pp. 400-409. 
neuentstehenden staatlichen Bürokratie und mit dem zentralistischen Parteiapparat, wobei sie unterliegen mussten. Die Sowjetdemokratie wurde durch die Parteibürokratie lahmgelegt. An die Stelle der Überwindung der Bürokratie trat ein - meist nur verbaler - Kampf gegen den Bürokratismus. Eine funktionierende Kontrolle des Staats- und Parteiapparates erwies sich nach Auflösung der Konstituante, nach Verbot konkurrierender Parteien, nach Aufhebung der Pressefreiheit und nach Verschmelzung der beiden bürokratischen Apparate als undurchführbar. Der charismatische Charakter der Sowjetbürokratie macht es unwahrscheinlich, daß sie sich freiwillig und kampflos in ein williges Instrument der Demokratie verwandeln lässt und es erlaubt, $\mathrm{da} ß$ ihre Funktionen auf das technisch erforderliche Maß reduziert werden. Die These Burnhams von der Analogie des Managerregimes in Ost und West ist eine Illusion. Der Glaube an die Entbehrlichkeit einer beamteten, aus Fachleuten bestehenden Verwaltung trug wesentlich zur Entstehung der totalitären Bürokratie mit bei. Die undifferenzierte Bürokratiefeindschaft wurde dem Bolschewismus zusammen mit Lenins Parteitheorie zum Verhängnis und so bewährte sich an den Dialektikern die Dialektik der Geschichte. 